\title{
Facteurs comportementaux et environnementaux associés au paludisme à Tourou (Bénin) en période de faible endémicité
}

\author{
Ibrahim MAMA CISSE ${ }^{1}$, Adébayo ALASSANI ${ }^{*}$, Mènonli ADJOBIMEY ${ }^{2}$, Rose \\ MIKPONHOUE $^{2}$, Antoine Vikkey HINSOU ${ }^{2}$ et Paul AYELO ${ }^{2}$
}

\author{
${ }^{1}$ Département de Médecine et Spécialités Médicales, Faculté de Médecine, Université de Parakou, Bénin. \\ ${ }^{2}$ Département de Médecine et Spécialités Médicales, Faculté des Sciences de la Santé, Université d'Abomey- \\ Calavi, Bénin. \\ *Auteur correspondant; E-mail: adebayoalassani@ gmail.com ; Tél.( 00229)95848436
}

\begin{abstract}
RESUME
Le paludisme demeure un problème de santé publique au Bénin malgré l'utilisation des moustiquaires. La présente étude avait pour objectif de déterminer la prévalence du paludisme à Tourou et d'identifier les facteurs comportementaux et environnementaux associés en période de faible endémicité. Il s'agissait d'une étude transversale, descriptive à visée analytique menée dans la population de Tourou. Le diagnostic du paludisme était fait par un test de diagnostic rapide. L'analyse des données a été faite par le logiciel SPSS version 21. Les facteurs de risque du paludisme ont été déterminés par régression logistique. Une p inférieure à 0,05 a été considérée comme significative. Au total 390 sujets ont été inclus dans l'étude. Une prédominance féminine $(50,6 \%)$ est observée avec un sex-ratio de 0,97. Sur les 390 sujets, 145 étaient infectés par le paludisme soit prévalence du $37,4 \%$. Le fait de dormir dehors (OR : 1,31) et après 23 heures (OR : 5,12) étaient des facteurs comportementaux à risque du paludisme tandis que l'absence d'eaux stagnantes $(\mathrm{OR}: 0,67)$ et celle des mauvaises herbes (OR : $0,67)$ étaient les facteurs environnementaux protecteurs contre le paludisme. La présente étude a montré que les facteurs environnementaux et comportementaux sont associés au paludisme dans la localité de Tourou. La lutte contre le paludisme devra considérer en plus de l'éradication du vecteur l'assainissement de l'environnement et le changement du mode de vie.
\end{abstract}

(C) 2020 International Formulae Group. All rights reserved.

Mots clés : Paludisme, Prévalence, Facteurs associés, Bénin.

\section{Behavioral and environmental factors associated with malaria in Tourou (Benin) during periods of low endemicity}

\begin{abstract}
Malaria remains a public health problem in Benin despite the use of mosquito nets. The objective of the present study was to determine the prevalence of malaria in Tourou and to identify behavioral and environmental factors associated to malaria in times of low endemicity. This was a cross-sectional, descriptive, analytical study carried out in the population of Tourou. The diagnosis of malaria was made by a rapid diagnostic test. Data analysis was done by SPSS version 21 software. Factors risk factor with malaria was determined by logistic regression. A p less than 0.05 was considered significant. A total of 390 subjects were included in the study. A female predominance $(50.6 \%$ ) was observed with a sex ratio of 0.97 . Of the 390 subjects, 145 were infected with malaria and the prevalence was $37.4 \%$. Sleeping outside (OR: 1.31) and after 11 p.m. (OR: 5.12) were behavioral
\end{abstract}


risk factors for malaria, while the absence of standing water (OR: 0.67) and that of weeds (OR: 0.67) were the protective environmental factors against malaria. The present study has shown that environmental and behavioral factors were associated with malaria in the locality of Tourou. The fight against malaria will have to consider in addition to the eradication of the vector the sanitation of the environment and the change of lifestyle.

(C) 2020 International Formulae Group. All rights reserved.

Keys words: Malaria, prevalence, associated factors, Benin.

\section{INTRODUCTION}

Le paludisme constitue l'une des principales causes de morbidité et de mortalité dans le monde surtout en Afrique au sud du Sahara (Yandaï et al., 2016; Rudasingwa et al., 2020; Solomon et al., 2020). Selon les dernières données publiées en décembre 2019, 228 millions de cas de paludisme sont recensés dans le monde en 2018 avec 405.000 décès (Bekono et al., 2020). L'Afrique au Sud du Sahara enregistre $88 \%$ des cas de paludisme et $90 \%$ des décès sur le plan mondial (Yadouleton et al., 2018; Fedaku et al., 2020). Les dépenses mondiales liées au paludisme sont énormes et croissantes allant de 960 millions de dollar en 2005 à 2,5 milliards en 2014 (Bah, 2020). Il s'agit d'une pathologie causée par cinq agents pathogènes (Doutoum et al., 2019) dont le Plasmodium falciparum qui est le plus responsable des cas graves de paludisme et le plus fréquemment rencontré en Afrique de l'Ouest (Bekono et al., 2020). Certains groupes de population sont plus à risque de paludisme et sont plus exposés à ses conséquences parmi lesquels on cite les enfants de moins de 5 ans, les gestantes, les personnes vivant avec le VIH, les sujets en visite dans les pays endémiques (Ukibé et al., 2019; Abossie et al., 2020). La fréquence, la sévérité et l'issue du paludisme sont influencées par les facteurs sociodémographique, environnemental, économique, culturel et comportemental (Hasyim et al., 2019). Ceci a motivé les autorités a accordé plus d'attention au paludisme avec l'adoption d'un certain nombre de mesures préventives parmi lesquelles l'utilisation des moustiquaires imprégnées d'insecticides et la pulvérisation d'insecticides avec comme corollaire une réduction de son incidence de $40 \%$ depuis l'an 2000 (Tusting et al., 2017). Le Bénin a adopté l'utilisation des moustiquaires imprégnées d'insecticides à longue durée d'action et des campagnes se font régulièrement pour sa distribution gratuite sur toute l'étendue du territoire. Cependant malgré ces mesures, le paludisme pose toujours un problème de santé publique et demeure la principale cause de morbi-mortalité. La nécessité d'adopter d'autres moyens de lutte s'avère indispensable vue l'insuffisance des mesures adoptées et aussi la résistance de plus en plus fréquente des vecteurs aux insecticides comme le dichlorodiphényltrichloéthane (Bekono et al., 2020). Pour ce faire, il paraît nécessaire d'agir sur d'autres facteurs favorisants notamment l'environnement et les comportements que la population adopte. Pour être efficace, l'identification des facteurs environnementaux et comportementaux associés au paludisme s'avèrent indispensables (Bekono et al., 2020). Peu de données existent au Bénin sur les facteurs comportementaux et environnementaux favorisant la survenue du paludisme. L'objectif de cette étude était de déterminer la prévalence du paludisme Tourou et d'identifier les facteurs comportementaux et environnementaux associés en période de faible endémicité

\section{MATERIEL ET METHODES}

\section{Type et période d'étude}

Il s'est agi d'une étude transversale descriptive et analytique dont la collecte des données a couvert la période allant du 20 février au 5 mars 2019. 


\section{Population d'étude}

L'étude s'est déroulée dans l'agglomération de Tourou, une localité du premier arrondissement de la commune de Parakou, la métropole du Nord Bénin. Elle est située à $7 \mathrm{~km}$ au Nord Ouest de la ville de Parakou, la plus grande métropole du nord Bénin. La population d'étude était constituée des habitants de la localité Tourou. Les sujets des deux sexes, de tout âge habitants la localité depuis au moins 6 mois et ayant donné leur consentement ont été inclus dans l'étude. Les sujets incapables de répondre aux questions n'y ont pas été inclus dans l'étude. Pour les sujets sont âgés de moins de 15 ans, le consentement a été obtenu après de l'un de ses parents et la collecte des données est faite chez les parents en dehors du test de diagnostic du paludisme.

\section{Échantillonnage}

La taille de l'échantillon a été calculée par la formule de Schwartz. La technique de sondage aléatoire en grappe à 4 degrés a été utilisée.

Calcul de la taille de l'échantillon

$\mathbf{n}=\frac{\varepsilon^{2} * p * q}{i^{2}}=\frac{(1,96)^{2} * 0,425 *(1-0,425)}{0,05^{2}}=375,5$ soit une taille minimale de 376 personnes

$>\mathrm{n}=$ effectif minimal attendu

$>\quad \varepsilon=1,96$ (écart réduit correspondant au risque d'erreur $\alpha$ à $5 \%$ )

$>\mathrm{p}=42,5 \%$ (prévalence du paludisme dans les départements de l'Alibori et du Borgou en 2016).

$$
\begin{aligned}
& >\quad \mathrm{q}=1-\mathrm{p} \\
& \text { résultats) } \mathrm{i}=0,05 \text { (précision souhaitée pour nos } \\
& >\quad \mathrm{k}=1 \text { (effet grappe) }
\end{aligned}
$$

Description de la technique de sondage aléatoire à 4 degrés

Premier degré : choix des grappes dans les quartiers: La base du sondage est constituée par la liste des 6 quartiers de Tourou établis avec leurs populations respectives; 30 grappes ont été choisis par défaut. La taille (W) de chaque grappe est sensiblement égale à $\mathrm{n} / 30$ ( $\mathrm{n}$ : le nombre de personnes à enquêter) individus par grappe soit $376 / 30=12,53$ arrondis à 13 .

Un nombre d est choisi au hasard entre 1 et le pas de grappe et a servi de base à l'identification de la première grappe à partir de la liste des populations cumulées. Au nombre d, on ajoute chaque fois le pas de grappes $\mathrm{k}$ pour obtenir autres grappes.

$\mathrm{k}=\mathrm{N} / 30$ soit $22003 / 30=734$ avec $\mathrm{N}$ est la population du village de Tourou.

Deuxième degré : Tirage des concessions. Dans chaque quartier, l'enquêteur est placé au centre du quartier et choisit au hasard une direction par la méthode de stylo tourné. Dans cette direction, il rentre dans une concession sur deux en commençant par le côté droit. La première concession à visiter est la deuxième dans la direction choisie.

Troisième degré : sélection des ménages par concession. Au sein de chaque concession retenue, des numéros sont attribués à chaque ménage sur des bouts de papier. Un ménage est tiré au sort.

Quatrième degré : sélection d'un individu par ménage. Parmi les ménages retenus et interrogés, un membre éligible de ce ménage tiré au sort. Il bénéficie d'un test de diagnostic rapide utilisant les antigènes solubles (HRPII) de Plasmodium falciparum.

N.B : Cas spécifique. Quand le nombre d'individus prévu n'est pas atteint dans une direction, l'enquêteur revient au centre du quartier et marche dans la direction opposée à la première en constituant l'échantillon de la même façon; quand la concession tirée ne comporte aucun ménage éligible, on procède à un nouveau tirage aléatoire parmi les concessions non tirées.

\section{Variables}

La variable dépendante étudiée est le paludisme diagnostiqué par le test de diagnostic rapide (TDR). Ainsi, dans cette étude, trois modalités ont été retenues pour cette variable à savoir : positif, négatif et indéterminé. Seuls ceux des résultats qui ont été positifs sont 
considérés comme ayant le paludisme. Les sujets sous traitement anti-palustre prescrit par un agent de santé sont considérés comme ayant un TDR positif. Les enquêtés dont le TDR est positif sont orientés vers le centre de santé du village. Il en est de même pour tous ceux qui ont une fièvre ou une altération de l'état général même si le TDR est négatif (ceux-ci ne sont pas considérés comme ayant souffrant d'un paludisme). Les variables indépendantes sont relatives aux données sociodémographiques, comportementales et environnementales.

\section{Collecte des données}

La collecte des données a été faite par l'entrevue face à face pour les données sociodémographiques à l'aide d'une fiche de collecte de données puis la réalisation du TDR pour le diagnostic du paludisme et l'observation de l'environnement immédiat pour les données environnementales.

\section{Analyse des données}

Les données recueillies ont été saisies et traitées avec le logiciel Spss version 21. L'apurement de la base a été fait avec la vérification de la complétude et de la cohérence des données. Les variables quantitatives sont exprimées en moyenne avec leur écart type. La regression logistique a été utilisée pour faire les analyses uni et multivariée. Une $\mathrm{p}<0,05$ a été considérée comme statistiquement significative.

\section{Aspects éthiques}

La confidentialité des données est respectée lors de l'enquête. Après avoir expliqué aux individus le but du travail, ils étaient libres de participer ou de ne pas participer à l'enquête et qu'une fois qu'ils ont accepté, elles étaient en droit de se désister après. L'anonymat est requis sur les fiches d'enquête. L'autorisation du chef du village et celle du chef de l'arrondissement ont été obtenues.

\section{RESULTATS}

Au total 390 sujets ont été inclus dans l'étude. Une prédominance féminine $(50,6 \%)$ était observée avec un sex-ratio de 0,97. L'âge moyen des enquêtés était d'environ $29 \pm 3,11$ ans avec les extrêmes de 1 an à 70 ans; $76,1 \%$ ont été sensibilisés sur le paludisme dont la connaissance est bonne chez $63,5 \%$. La fièvre est retrouvée chez $24,9 \%$ et $31,5 \%$ avaient une altération de l'état général. Sur le plan comportemental, $66,5 \%$ utilisaient des insecticides, $87,4 \%$ dormaient sous moustiquaire, $68,4 \%$ dormaient hors des chambres et 79,4\% dormaient avant 23 heures. L'environnement était caractérisé par la présence des eaux stagnantes dans $44,8 \%$, l'habitat proche d'un cours d'eau dans $22,9 \%$ et la présence des mauvaises herbes dans $93,6 \%$ (Tableau 1).

Sur les 390 sujets, 145 étaient infectés par le paludisme soit prévalence de $37,4 \%$ (Figure 1). Parmi ceux-ci, 11(7,58\%) étaient connus et sous traitement antipalustre. Parmi les sujets infectés, $34(23,44 \%)$ présentaient une fièvre et $27(18,62 \%)$ une altération de l'état général.

En analyse univariée, les facteurs de risque du paludisme étaient le fait de dormir dehors, dormir après 23 heures, la présence d'eaux stagnantes et celle des mauvaises herbes (Tableau 3).

En multivariée, le fait de dormir dehors $(\mathrm{OR}: 1,31 ; \mathrm{p}=0,000)$ et après 23 heures (OR : 5,12; $\mathrm{p}=0,000)$ étaient des facteurs comportementaux à risque du paludisme tandis que l'absence d'eaux stagnantes (OR: 0,67; $\mathrm{p}=0,000)$ et celle des mauvaises herbes (OR : $0,67 ; \quad \mathrm{p}=0,001)$ étaient les facteurs environnementaux protecteurs contre le paludisme (Tableaux 2 et 3). 
Tableau 1: Caractéristiques sociodémographiques, comportementales et environnementales de la population d'étude $(\mathrm{n}=390)$.

\begin{tabular}{|c|c|c|}
\hline & $\mathrm{n}$ & $\%$ \\
\hline \multicolumn{3}{|c|}{ Caractéristiques sociodémographiques } \\
\hline \multicolumn{3}{|l|}{ Age (ans) } \\
\hline$\geq 5$ ans & 280 & 72,0 \\
\hline \multicolumn{3}{|l|}{ Sexe } \\
\hline Féminin & 197 & 50,6 \\
\hline \multicolumn{3}{|l|}{ Taille du ménage } \\
\hline$\leq 5$ & 221 & 56,8 \\
\hline \multicolumn{3}{|l|}{ Type de ménage } \\
\hline Monagame & 201 & 51,6 \\
\hline \multicolumn{3}{|l|}{ Type de famille } \\
\hline Biparentale & 322 & 82,5 \\
\hline \multicolumn{3}{|c|}{ Niveau socio-économique } \\
\hline Bon & 266 & 68,1 \\
\hline \multicolumn{3}{|c|}{ Niveau d'instruction } \\
\hline Aucun & 179 & 45,8 \\
\hline \multicolumn{3}{|c|}{ Sensibilisation sur le paludisme } \\
\hline Oui & 297 & 76,1 \\
\hline \multicolumn{3}{|c|}{ Connaissance sur le paludisme } \\
\hline Bonne & 248 & 63,5 \\
\hline \multicolumn{3}{|l|}{ Présence de fièvre } \\
\hline Oui & 97 & 24,9 \\
\hline \multicolumn{3}{|l|}{ Etat général } \\
\hline Altéré & 123 & 31,5 \\
\hline \multicolumn{3}{|c|}{ Caractéristiques comportementales } \\
\hline \multicolumn{3}{|l|}{ Usage d'insecticide } \\
\hline Oui & 259 & 66,5 \\
\hline \multicolumn{3}{|c|}{ Utilisation de moustiquaire } \\
\hline Oui & 341 & 87,4 \\
\hline \multicolumn{3}{|l|}{ Lieu de sommeil } \\
\hline Hors des chambres & 267 & 68,4 \\
\hline \multicolumn{3}{|l|}{ Heure de sommeil } \\
\hline Avant $23 \mathrm{H}$ & 210 & 79,4 \\
\hline \multirow{2}{*}{\multicolumn{3}{|c|}{ Caractéristiques environnementales }} \\
\hline \multicolumn{2}{|c|}{ Présence d'eaux stagnantes } & \\
\hline Oui & 175 & 44,8 \\
\hline \multicolumn{3}{|c|}{ Habitat proche d'un cours d'eau } \\
\hline Oui & 89 & 22,9 \\
\hline \multicolumn{3}{|c|}{ Présence de mauvaises herbes } \\
\hline Oui & 365 & 93,6 \\
\hline
\end{tabular}




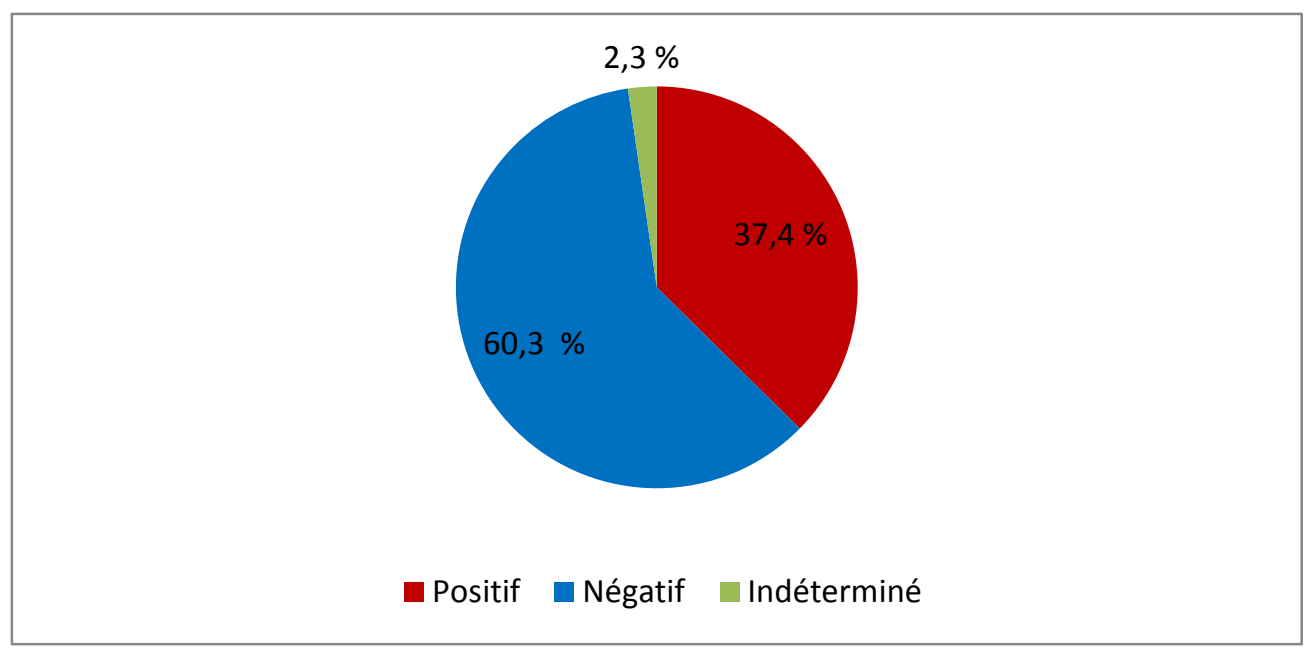

Figure 1 : Prévalence du paludisme à Tourou.

Tableau 2 : Facteurs comportementaux et environnementaux associés au paludisme à Tourou (analyse univariée).

\begin{tabular}{|c|c|c|c|}
\hline \multicolumn{4}{|c|}{ Paludisme } \\
\hline & Oui $(\%)$ & OR (IC à 95\%) & $\mathbf{p}$ \\
\hline \multicolumn{4}{|c|}{ Facteurs comportementaux } \\
\hline \multicolumn{4}{|c|}{ Usage d'insecticide } \\
\hline Oui & 25,8 & 1 & 0,171 \\
\hline Non & 11,6 & $0,37[0,27 ; 0,89]$ & \\
\hline \multicolumn{4}{|c|}{ Utilisation de moustiquaire } \\
\hline Oui & 30 & 1 & 0,158 \\
\hline Non & 7,41 & $0,15[0,087 ; 0,0,53]$ & \\
\hline \multicolumn{4}{|l|}{ Lieu de sommeil } \\
\hline Chambre & 17,74 & 1 & $\mathbf{0 , 0 0 0}$ \\
\hline Dehors & 19,67 & $1,27[1,13 ; 1,77]$ & \\
\hline \multicolumn{4}{|c|}{ Heure de sommeil } \\
\hline Avant $23 \mathrm{H}$ & 6,12 & 1 & 0,009 \\
\hline A partir de $23 \mathrm{H}$ & 31,29 & $5,23[2,03 ; 9,37]$ & \\
\hline \multicolumn{4}{|c|}{ Facteurs environnementaux } \\
\hline \multicolumn{4}{|c|}{ Présence d'eaux stagnantes } \\
\hline Oui & 23,22 & 1 & $\mathbf{0 , 0 0 0}$ \\
\hline Non & 14,19 & $0,73[0,49 ; 0,76]$ & \\
\hline \multicolumn{4}{|c|}{ Habitat proche d'un cours d'eau } \\
\hline Oui & 9,35 & 1 & 0,064 \\
\hline Non & 49,03 & $4,79[3,36 ; 7,12]$ & \\
\hline \multicolumn{4}{|c|}{ Présence de mauvaises herbes } \\
\hline Oui & 36,77 & 1 & 0,0087 \\
\hline Non & 3,23 & $0,15[0,11 ; 0,51]$ & \\
\hline
\end{tabular}


Tableau 3 : Facteurs comportementaux et environnementaux associés au paludisme à Tourou (analyse multivariée).

\begin{tabular}{lllc}
\hline & \multicolumn{1}{c}{ Paludisme } \\
\hline & Oui (\%) & OR (IC à 95\%) & p \\
\hline Facteurs comportementaux & & & \\
\hline Lieu de sommeil & 16,34 & 1 & $\mathbf{0 , 0 0 0}$ \\
$\begin{array}{l}\text { Chambre } \\
\text { Dehors }\end{array}$ & 19,97 & $1,31[1,03 ; 1,67]$ & \\
Heure de sommeil & & & \\
Avant 23H & 5,82 & 1 & $\mathbf{0 , 0 0 0}$ \\
A partir de 23H & 30,77 & $5,12[3,07 ; 8,17]$ & \\
$\begin{array}{l}\text { Facteurs environnementaux } \\
\text { Présence d'eaux stagnantes }\end{array}$ & & & \\
Oui & 25,12 & 1 & $\mathbf{0 , 0 0 0}$ \\
Non & 13,29 & $0,67[0,49 ; 0,86]$ & \\
Présence de mauvaises herbes & & & $\mathbf{0 , 0 0 1}$ \\
Oui & 33,75 & 1 & \\
Non & 4,77 & $0,12[0,09 ; 0,48]$ & \\
\hline
\end{tabular}

\section{DISCUSSION}

La présente étude a permis de rapporter une prévalence du paludisme de $37,4 \%$. Une prévalence qui devrait être plus importante si l'étude a été menée en saison pluvieuse, période de forte endémie palustre. Ramdzan et al. (2020) en Indonésie et Meresa et al. (2018) en Ethiopie ont rapporté des prévalences similaires respectivement $33,6 \%$ et $45,3 \%$ en population. Une prévalence plus élevée a été rapportée par Tusting et al. (2017) au Burkina Faso notamment $70,6 \%$ à l'examen microscopique et $79,8 \%$ au test diagnostic rapide. Parmi les sujets infectés de l'étude, 23,44\% étaient symptomatiques. Dans l'étude de Mbohou et al. (2019) au Cameroun, 95,71\% des sujets infectés ne présentent aucun signe de maladie. Le fait que le paludisme soit asymptomatique dans la majorité des cas ne permet pas de prendre des mesures curatives et surtout préventives; l'anophèle pouvant faire passer le parasite d'un sujet infecté asymptomatique ou non vers un sujet sain. Pour réduire la fréquence du paludisme, il est important d'agir sur les facteurs favorisants.

Le fait de dormir dehors et après 23 heures sont les facteurs comportementaux à risque du paludisme dans le présent travail. Plusieurs études ont abouti aux résultats similaires. C'est le cas des études de Agegnehu et al. (2018) et de Fedaku et al. (2020) qui soulignent que le fait de dormir dehors est associé au paludisme. Tetelepta et al. (2019) en Indonésie rapporte que les sujets ayant d'habitude de rester hors des chambres sont plus à risque de paludisme. Pour Mosnier et al. (2020) les personnes effectuant les travaux nocturnes sont plus à risque de paludisme. Ces différentes associations s'expliquent par le fait que les piqûres de moustique s'observent le plus souvent le soir. Pour ce fait, les sujets dormant dehors ou tard doivent adopter des attitudes comme le port de vêtements longs ou la pulvérisation des insecticides afin d'éviter les piqûres des moustiques.

Parmi les facteurs environnementaux, la présence d'eaux stagnantes et celle des mauvaises herbes étaient des facteurs à risque du paludisme dans notre étude. La proximité des eaux stagnantes comme facteur favorisant la survenue du paludisme est rapportée par Hailu et al. (2018), Gopal et al. (2019) et Dufera et al. (2020). Dans l'étude de Abiodun et al. (2018), la survenue du paludisme est fonction de l'index de la végétation. Plus il y existe la présence de mauvaise, plus le paludisme est fréquent. Le risque de transmission du paludisme dépend de la distance de vol de l'anophèle qui est approximativement de 2 à 3 $\mathrm{km}$. Les habitats se trouvant à moins de $250 \mathrm{~m}$ 
de la forêt ou $200 \mathrm{~m}$ des jardins sont plus à risque de paludisme (Rahayu et al., 2019). Ceci implique la nécessité de maintenir sain l'environnement en évitant la présence des eaux stagnantes et en dégageant les mauvaises herbes dans les concessions. La pulvérisation des insecticides dans les eaux stagnantes ou les herbes peut être aussi un moyen de prévention.

\section{Conclusion}

facteurs

La présente étude a montré que les comportementaux sont associés au paludisme dans la localité de Tourou. La lutte contre le paludisme devra considérer en plus de l'éradication $\mathrm{du}$ vecteur mais aussi l'assainissement de l'environnement et le changement du mode de vie. Un accent particulier doit être mis sur l'éviction des mauvaises herbes et des eaux stagnantes ainsi que le sommeil dehors et tardif.

\section{CONFLIT D'INTERETS}

Les auteurs déclarent qu'ils n'ont aucun conflit d'intérêts pour cet article.

\section{CONTRIBUTIONS DES AUTEURS}

IMC a initié le travail; AA a écrit le manuscrit; MA a réalisé les statistiques; RM, AVH, PA ont lu et corrigé le manuscrit.

\section{REMERCIEMENTS}

A l'endroit de la population de Tourou pour avoir accepté participer à l'étude.

\section{REFERENCES}

Solomon A, Kahase D, Alemayehu. 2020. Trend of malaria prevalence in Wolkite health center: an implication towards the elimination of malaria in Ethiopia by 2030. Malaria Journal, 19: 112-119.

Rudasingwa G, Cho S. 2020. Determinants of the persistence of malaria in Rwanda. Malaria Journal, 19: 36-44. DOI: https://doi.org/10.1186/s12936-02003182-z

Yandaï FH, Moundine K, Djoumbé E, Boulotigam K, Moukenet A, Demba Koudino, Kerah Hinzoumbé C. 2016. Perception de risques du paludisme et utilisation des moustiquaires au Tchad. Int. J. Biol. Chem. Sci., 10(6): 2646-2654. DOI:

http://dx.doi.org/10.4314/ijbcs.v10i6.19
Bekono B, Ntie-kang F, Onguéné P, Lifongo L, Sippl W, Fester K. 2020. The potential of anti-malarial compounds derived from African medicinal plants: a review of pharmacological evaluations from 2013 to 2019. Malaria Journal, 19: 183-217. DOI : $\quad$ https://doi.org/10.1186/s12936020-03231-7

Fedaku M, Yenit M, Lakew A. 2020. The prevalence of asymptomatic malaria parasitemia and associated factors among adults in Dembia district, northwest Ethiopia, 2017. Archives of Public Health, 76: 74-79. DOI: https://doi.org/10.1186/s13690-0180323-z

Yadouleton A, Aïkpon R, Houndéton G, Aboubakar S, Ursins F, Tchibozo C, Agolinou A, Akogbéto M. 2018. Données entomologiques préliminaires pour la mise en place d'une pulvérisation intradomiciliaire à grande échelle dans la commune de Corpargo au Nord-Est du Bénin. Int. J. Biol. Chem. Sci., 12(5): 1993-2003.

DOI: https://dx.doi.org/10.4314/ijbcs.v12i5.3

Bah MS. 2020. The Relationship Between Malaria Status in Under-five Children and Some Household Demographic, Socioeconomic and Environmental Factors Associated with the Disease in Sierra Leone. Thesis, Georgia State University, Georgia. https://scholarworks.gsu.edu/iph_theses/7 06

Doutoum AA, Moundine K, Djoumbé E, Boulotigam K, Moukenet Azoukalne, Demba Kodindo I, Kerat Hinzoumbé C. 2019. Perception de risques du paludisme et utilisation des moustiquaires au Tchad. Int. J. Biol. Chem. Sci., 10(6): 2646-2654. DOI: http://dx.doi.org/10.4314/ijbcs.v10i6.19

Abossie A, Yohanes T, nedu A, Tafesse W, Damitie M. 2020. Prevalence of Malaria and Associated Risk Factors Among Febrile Children Under Five Years: A Cross-Sectional Study in Arba Minch Zuria District, South Ethiopia. Infection and Drug Resistance;13: 363-372 http://doi.org/10.2147/IDR.S223873

Ukibé NR, Onyénékwé C, Anojulu A, Onwubuya E, Kalu O, Ukibé S. 2019. Impact of Plasmodium falciparum malaria infection on serum cortisol, 
adrenocorticotropic hormone, pregnancy associated plasma protein-A and alphafetoprotein in pregnant women at Nnewi. Int. J. Biol. Chem. Sci., 13(3): 1222-1230. DOI:

https://dx.doi.org/10.4314/ijbcs.v13i3.1

Hasyim H, Dale P, Groneberg D, Kuch U, Müller R. 2019. Social determinants of malaria in an endemic area of Indonesia. Malaria Journal, 18: 134-144. DOI: https://doi.org/10.1186/s12936-0192760-8

Tusting LS, Bottomley C, Gibson H, Kleinschmidt I, Tatem AJ, Lindsay SW, Gething PW. 2017. Housing Improvements and Malaria Risk in SubSaharan Africa: A Multi-Country Analysis of Survey Data. PLoS Med., 14(2): e1002234. DOI: https://doi:10.1371/journal.pmed.100223 4

Ramdzan AR, Ismail A, Mohd Zanib ZS. 2020. Prevalence of malaria and its risk factors in Sabah, Malaysia. International Journal of Infectious Diseases, 91: 68-72. DOI: https://doi.org/10.1016/j.ijid.2019.11.026

Meresa S, Megbaru A, Kiros T, Tadesse D, Bayissa S, Bugssa G. 2018. The Prevalence of Malaria in Tselemti Wereda, North Ethiopia: A Retrospective Study. Ethiop. J. Health Sci., 28(5): 539546. http://dx.doi.org/10.4314/ejhs.v28i5.4

Mbohou CN, Foko LPK, Nyabeyeu HN, Tonga C, Nono LK, Kangam L, Bunda GW, Mbou IM, Hondt EON, Mbe AJK, Nolla NP, Lehman LG. 2019. Malaria screening at the workplace in Cameroon. PLOS ONE, 14(12): e0225219. DOI: https://doi.org/10.1371/journal.pone.0225 219

Agegnehu F, Shimeka A, Berihun F, Tamir M. 2018. Determinants of malaria infection in Dembia district, Northwest Ethiopia: a case-control study. BMC Public Health, 18: 480-487. DOI: https://doi.org/10.1186/s12889-0185370-4

Tetelapta M, Raharjo M, Nurjazuli L. 2019. Variability of Environmental Quality and Dynamics of Malaria in Sarmi District. International Journal of English, Literature and Social Sciences, 4(6):
1845-1849.

https://dx.doi.org/10.22161/ijels.46.36

Mosnier E, Roux E, Cropet C, Lazrek Y, Moriceau O, Gaillet M. 2020. Prevalence of Plasmodium spp. in the Amazonian Border Context (French Guiana-Brazil): Associated Factors and Spatial Distribution. Am. J. Trop. Med. Hyg., 102(1): 130-141. DOI: https://dx.doi:10.4269/ajtmh.19-0378

Hailu T, Alemu M, Mulu W, Abera B. 2018. Incidence of Plasmodium infections and determinant factors among febrile children in a district of Northwest Ethiopia; a cross-sectional study. Tropical Diseases, Travel Medicine and Vaccines, 4: 8-13. DOI: https://doi.org/10.1186/s40794-0180069-1

Gopal S, Ma Y, Xin C, Pitts J, Were L. 2019. Characterizing the Spatial Determinants and Prevention of Malaria in Kenya. Int. J. Environ. Res. Public Health, 16 : 5078 5096.

DOI : https://doi:10.3390/ijerph16245078

Dufera M, Dabsu R, Tiruneh G. 2020. Assessment of malaria as a public health problem in and around Arjo Didhessa sugar cane plantation area, Western Ethiopia. BMC Public Health, 20: 655664.

DOI: https://doi.org/10.1186/s12889-02008784-5

Abiodun G, Adebiyi B, Abiodun R, Oladimeji O, Oladimeji K, Adeola A, Makinde OS, Okosun KO, Djidjou-Demasse R, Semegni YJ, Njabo KY, Witbooi PJ, Aceves A. 2020. Investigating the Resurgence of Malaria Prevalence in South Africa Between 2015 and 2018: A Scoping Review. The Open Public Health Journal, 13: 119-125. https://doi 10.2174/1874944502013010119

Rahayu S, Handayani O, Ngaga L, Sudana I, Budiono I. 2019. The Effects of Rumah Panggung Environment, Social Culture, and Behavior on Malaria Incidence in Kori Village, Indonesia. World Academy of Science, Engineering and Technology. International Journal of Medical and Health Sciences, 13(9): 395-399. DOI: https//doi.waset.org/Publication/1001072 8. 